\title{
La cultura financiera en estudiantes de administración y comunicación de la Universidad Nacional Jorge Basadre Grohmann-Perú, 2018
}

\author{
The financial culture in administrative and communication students of \\ the national university Jorge Basadre Grohmann-Peru, 2018
}

\section{${ }^{1}$ Juan Apaza Paucara}

\section{RESUMEN}

El objetivo de esta investigación fue determinar el nivel de conocimiento sobre la cultura financiera de los estudiantes del VI y VIII ciclo de la Escuela Profesional de Administración y del II ciclo de la Escuela Profesional de Ciencias de la Comunicación de la Universidad Nacional Jorge Basadre Grohmann de Tacna, Perú. La cultura financiera es un tema económico fundamental para todas las organizaciones internacionales; este proceso de concienciación ha advertido la necesidad de desarrollar y profundizar las capacidades financieras de la población mundial, orientando las decisiones financieras de las personas. El tipo de investigación fue básico y el diseño, no experimental. La muestra estuvo conformada por un grupo de 90 estudiantes (de ambas escuelas profesionales). El tipo de instrumento elegido fue el cuestionario, con una fiabilidad de alfa de Cronbach de 0.903 . Se concluyó que, con respecto al nivel de conocimiento sobre la cultura financiera de los estudiantes de ambas escuelas, el $75.56 \%$ obtuvo un nivel alto y el $2.22 \%$, un nivel bajo. Además, con respecto a las dimensiones que comprende la cultura financiera; respecto la dimensión crédito se halló que un $54.4 \%$ logró un nivel alto y el $3.3 \%$, un nivel bajo. En cuanto a la dimensión de seguros, el $65.6 \%$ obtuvo un nivel medio y el $4.4 \%$, un nivel bajo. En la descripción sobre la dimensión medios de pago, el $82.2 \%$ obtuvo un nivel medio y el $7.8 \%$, un nivel bajo. El hallazgo en la dimensión tasa de interés destacó que el $61.1 \%$ obtuvo un nivel alto y el $5.6 \%$, un nivel bajo. El conocimiento sobre la dimensión presupuesto destacó que el $70 \%$ obtuvo un nivel alto y el $3.3 \%$, un nivel bajo. Se ha determinado el conocimiento sobre la dimensión ahorro resultado que el $60 \%$ obtuvo un nivel alto y un $5.6 \%$, un nivel bajo. Finalmente, el hallazgo sobre conocimiento de la dimensión de inversión fue el $63.3 \%$ alcanzó un nivel alto y un $4.4 \%$, un nivel bajo.

Palabras Clave: Conocimiento, la cultura financiera, riesgo.

\section{ABSTRACT}

The objective of this research was to determine the level of knowledge about the financial culture of the sixth and eighth cycle students of the Professional School of Administration and the second cycle of the Professional School of Communication Sciences of the National University Jorge Basadre Grohmann. Tacna, Peru Financial culture is a fundamental economic issue for all international organizations; This process of awareness has warned of the need to develop and deepen the financial capabilities of the world population, guiding the financial decisions of the people. The type of research was basic and the design, not experimental. The sample consisted of a group of 90 students (from both professional schools). The type of instrument chosen was the questionnaire, with a reliability of Cronbach's alpha of 0.903. It was concluded that, regarding the level of knowledge about the financial culture of the students of both schools, $75.56 \%$ obtained a high level and $2.22 \%$, a low level. In addition, with respect to the dimensions that comprise the financial culture; Regarding the credit dimension, it was found that $54.4 \%$ achieved a high level and $3.3 \%$, a low level. Regarding the dimension of insurance, $65.6 \%$ obtained an average level and $4.4 \%$, a low level. In the description on the means of payment dimension, $82.2 \%$ obtained a medium level and $7.8 \%$, a low level. The finding in the interest rate dimension highlighted that $61.1 \%$ obtained a high level and $5.6 \%$, a low level. Knowledge about the budget dimension highlighted that $70 \%$ obtained a high level and 3.3\%, a low level. The knowledge about the savings dimension has been determined that $60 \%$ obtained a high level and $5.6 \%$, a low level. Finally, the finding on knowledge of the investment dimension was $63.3 \%$ reached a high level and $4.4 \%$, a low level.

Keywords: Knowledge, financial culture, risk.

${ }^{1}$ Escuela Profesional de Ciencias Administrativas. Universidad Nacional Jorge Basadre Grohmann. Tacna-Perú.

E-mail:japaza@unjbg.edu.pe

Presentado: 24/01/19, Aprobado: 31/05/19 


\section{INTRODUCCIÓN}

La investigación tuvo como objetivo de estudio determinar el nivel de conocimiento sobre la cultura financiera en los estudiantes de la Escuela Profesional de Administración (ESAD) y Escuela Profesional de Ciencias de la Comunicación (ESCC) de la Universidad Nacional Jorge Basadre Grohmann (UNJBG) de Tacna, Perú, realizada durante los meses de octubre y noviembre de 2018; cuya muestra estuvo conformada por 90 estudiantes de ambas escuelas profesionales de la UNJBG.

La cultura financiera, hoy en día, es reconocida como tema económico fundamental por todas las organizaciones internacionales. Este proceso de concienciación ha advertido la necesidad de desarrollar y profundizar las capacidades financieras de la población mundial, elevando su bajo nivel de cultura financiera; que se comprenda la responsabilidad de las personas sobre orientar sus decisiones financieras y que se conozca el proceso de sofisticación de los productos y mercados financieros. En América Latina, esto puede ser posible a partir del reconocimiento de las necesidades específicas de la población: sus actividades productivas y sus formas de intercambio monetarias y no monetarias. Es así cómo, para cubrir estas necesidades, se desarrollan modelos y programas de educación financiera, orientados a diversas poblaciones de acuerdo a sus condiciones sociales y económicas, cuyo propósito principal es el fortalecimiento de capacidades financieras de la población de bajos recursos. La adopción de dichos programas y modelos se establece por medio de políticas gubernamentales que se apoyan en los enunciados de educación financiera, propuestos por la Organización para la Cooperación del Desarrollo Económico (OCDE), los cuales son adaptados por cada país y puestos en práctica, teniendo en cuenta las condiciones particulares que los caracteriza. Adicionalmente, la Comisión Multisectorial de Inclusión Financiera (CMIF) (2015) señala que la educación financiera constituye una línea de acción fundamental para promover la inclusión financiera en el país. Es así que, los objetivos relacionados con la educación financiera están orientados a lograr mejoras en las capacidades, actitudes y conocimientos financieros de todos los segmentos de la población para la adecuada toma de decisiones económicas.

Todo ello lleva a formular la siguiente interrogante:

¿Cuáles son los conocimientos sobre cultura financiera de los estudiantes universitarios de Administración y Ciencias de la Comunicación?

\section{MARCOTEÓRICO}

Según Ardila y Rengifo (2014), el reconocimiento mundial de la Educación Financiera como un hecho económico importante es innegable. Habiendo sido esto examinado y declarado por las instituciones multilaterales y los foros internacionales como el de la OCDE, el Banco Mundial, el G-20, el Foro de Cooperación Económica Asia-Pacífico (APEC) y la Asociación de Naciones del Sudeste Asiático (ASEAN). Por ello, podemos afirmar que existe un mayor nivel de conciencia a nivel internacional. $Y$ esto ha sido posible a través de la constatación de los siguientes hechos: al bajo nivel de cultura financiera detectado por los organismos internacionales mediante las evaluaciones a los programas que están actualmente en ejecución, al reconocimiento de la responsabilidad de las personas para orientar sus decisiones financieras y al proceso de sofisticación de los productos y mercados financieros.

Cabrera y Zapata (2017) consideran importante la evaluación del conocimiento de la materia financiera de los estudiantes universitarios. Asimismo, señalan que la salud financiera es el resultado de la eficiente administración de los recursos monetarios, el uso controlado del financiamiento y el fomento al ahorro, a través de una serie de conductas que permiten a las personas controlar en sus gastos y planificar el cumplimiento de las obligaciones que contraen en materia económica. Por otro lado, la Comisión Multisectorial de Inclusión Financiera (2015), en su documento, precisa:

El Perú ha planteado retos para lograr mejoras sustanciales en materia de educación financiera, incrementar los niveles de bancarización, promover 
modelos de negocio que permitan reducir el "elevado costo de las transferencias y transacciones inherentes ligadas a los productos, sobre todo en zonas remotas" y finalmente, continuar con las políticas regulatorias y de supervisión que permitan mitigar el riesgo de sobreendeudamiento y faciliten los procesos de innovación para el desarrollo de nuevos productos financieros inclusivos. (p.10)

Quintero (2014), en su investigación, presentó una "propuesta de formación en educación económica y financiera desde la perspectiva del desarrollo humano". En esta propuesta, además señalar la posibilidad de desarrollar principios y valores a través de ella, sostiene que: "El bajo nivel de educación financiera en la mayoría de la población, tal y como lo plantean diferentes estudios realizados, incide de manera negativa en el bienestar de las comunidades y el desarrollo armónico de la economía de un país" (p.219).

Por otro lado, Briano (2016) reflexiona sobre el uso de la tarjeta de crédito como un medio de pago de obtención fácil en el sistema bancario $\mathrm{y}$, al mismo tiempo, los aspectos negativos que conlleva. Por ello señala que: "es un medio de pago globalmente aceptado, lo que lleva a incrementar el consumo y endeudamiento de las personas. De ahí la importancia de desarrollar las habilidades financieras para promover una mayor responsabilidad y uso adecuado del dinero" (p.5).

Entre los factores positivos de contar con una educación financiera, podemos mencionar, por ejemplo, que los jóvenes evitan o resuelven sus problemas financieros, lo cual incide en una vida más próspera, saludable y feliz.

Briano (2016) pone de manifiesto que problemas cotidianos como el divorcio, enfermedades mentales o infelicidad son causados en la mayoría de las ocasiones por problemas financieros. Del mismo modo, Cleek y Pearson (como se citó en Briano, 2016) señalan que, de acuerdo a una encuesta americana sobre las causas de divorcio se mostró que el $32.9 \%$ de las mujeres y el 28.7 \% de los hombres afirmaron que los problemas financieros fueron una de las razones principales de divorcio. Por ello, podemos afirmar, al igual Wolcott y Hughes (como se citó en Briano, 2016) que: "El impacto negativo en el matrimonio, los problemas financieros personales pueden llegar a causar estrés, depresión y baja autoestima, lo cual incide en las relaciones familiares" (p.6).

Sallie (como se citó en Lusardi, Mitchell, \& Curto, 2010) realizó una encuesta a los estudiantes universitarios sobre la educación financiera, donde obtuvo los siguientes resultados: el $84 \%$ de los estudiantes universitarios dijeron que necesitaban más educación sobre temas de gestión financiera al $64 \%$ les habría gustado recibir información sobre temas de administración financiera en la escuela secundaria, y al $40 \%$ le hubiera gustado recibir información como estudiantes de primer año de universidad. Por ello, es necesario destacar la importancia crítica para los políticos, en varios ámbitos, sobre cómo ayudar a diseñar una educación financiera efectiva de programas dirigidos a los jóvenes y también ayudar en una legislación para proteger a los consumidores jóvenes. Asimismo, Lusardi et al. (2010) reflexiona sobre la influencia que tiene la educación financiera en el comportamiento del consumidor. Menciona que las personas con baja educación financiera tienen más probabilidades de tener problemas con deudas, tienen menos probabilidades de participar en el mercado de valores, es menos probable que elijan fondos mutuos con tarifas más bajas, es menos probable que acumulen riqueza y administren la riqueza de manera efectiva y menos propensos a planificar para la jubilación

Para López (2016), es un círculo vicioso el bajo nivel de responsabilidad, por ello defiende que "el control financiero es una constante en todas las etapas de la vida". El reto se convierte en sentar una base académica en la temática referida a los jóvenes adultos en temas de planificación financiera personal para que de esta manera eleven sus habilidades en la gestión financiera personal, conocimiento $\mathrm{y}$ experiencia. Por otro lado, Flores (2018) en su investigación sostiene: "La educación 
financiera motiva el ahorro y la baja alfabetización ha conducido al uso de préstamos a corto plazo de soluciones familiares y en la amortiguación de situaciones de crisis financiera" (p.17). Además, que la buena preparación es una estrategia de largo plazo, de gestiones empresariales, jubilación, salud y emergencias de gastos médicos en el presente y en el futuro y para una mejor calidad de vida. Aylas (2017), en su investigación sobre cultura financiera en la Universidad Continental - sede Huancayo, concluye que el $78 \%$ de los estudiantes consideran que si una persona tiene conocimientos sobre educación financiera tomará buenas decisiones en cuanto al uso de la tarjeta de crédito, por otro lado, el conocimiento de temas financieros en los estudiantes se evidencia, ya que el $75 \%$ de ellos realizan presupuestos mensuales, lo cual demuestra que son capaces de tomar buenas decisiones.

Siguiendo otro concepto sobre la cultura financiera, Blancas (2016) afirma:

La cultura financiera es el proceso mediante el cual, tanto los consumidores como los inversionistas financieros logran un mejor conocimiento de los diferentes productos financieros, sus riesgos y beneficios, y que, mediante la información o instrucción, desarrollan habilidades que les permiten una mejor toma de decisiones, lo que deriva en un mayor bienestar económico. (p. 30)

Por otro lado, García (2017), con respecto al crédito comercial, señala:

El crédito comercial es una fuente de financiación alternativa a corto plazo y de naturaleza informal, cuyos términos en general, no están fijados de forma legal, sino que dependen de la industria y el país en que se apliquen. Estos créditos se los conceden entre sí las empresas no financieras y consiste en el aplazamiento en el pago que las empresas conceden a sus clientes en una transacción comercial de compraventa de bienes o servicios. (p.9)
Referente a la tasa de interés Blacio (2016) sostiene que:

Para poder tomar una decisión correcta frente a las ofertas que se nos pueda presentar para invertir nuestro dinero, no nos podemos dejarnos vislumbrar a simple vista por el rendimiento ofrecido sin tomar en cuenta la tasa de interés nominal y el tiempo a la que nuestro dinero estará en custodia de las instituciones financieras. (p. 16)

Otro término importante es el presupuesto. Al respecto, Burbano (como se citó en Fagilde, 2009) señala que el presupuesto "es la estimación programada, de manera sistemática, de las condiciones de operación y de los resultados a obtener por un organismo en un periodo determinado". También dice que el presupuesto "es una expresión cuantitativa formal de los objetivos que se propone alcanzar la administración de la empresa en un periodo, con la adopción de las estrategias necesarias para lograrlos" (p.6-8).

Larios (2009) hace referencia sobre el hábito del ahorro, menciona que este es considerado prioritario para el desarrollo de habilidades de organización, control y evaluación para el logro de objetivos y metas establecidas tanto en manera individual como en forma colectiva.

Según Lobato, Santillán y Hernández (2018), el ahorro es uno de los aspectos más importantes en la vida económica de las personas, debido a que es la clave para obtener una independencia financiera y acumular riqueza. Además, el tener ahorros permite una seguridad económica y el poder cumplir metas como poner un negocio propio o adquirir algún bien como una vivienda o un automóvil.

Respecto a la inversión, García (2014) hace referencia sobre las expectativas de mejora, de crecimiento de inversión, de ahorro y de solvencia financiera como temas tratados día a día por mucha gente y no solo por los expertos.

Por todo lo antes mencionado, sobre una buena educación financiera, es necesario resaltar su importancia. Tinoco (2017) señala:

Una buena educación financiera se convierte en una herramienta o instrumento importante para que el 
crédito en Perú no sea una necesidad permanente y que el endeudamiento no represente un estilo de vida; así como, enseñar que ahorrar e invertir, elaborar y administrar un presupuesto personal, genera riquezas para todo ciudadano en este país. Esta preocupación se instala como idea de investigación en la formación de los estudiantes universitarios del departamento de Junín, siendo ellos ciudadanos que demuestran comportamientos frente al ahorro, uso de tarjetas de crédito, sujetos de crédito, en la investigación se logró determinar que en el aspecto de planeación y control financiero no logra generar una aplicación en la vida de los estudiantes que garantice el buen manejo de sus finanzas personales (p.36).

Por otro lado, en los estudios de García, Grifoni, López y Mejía (2013) sobre la educación financiera en América Latina y el Caribe se señala que la situación actual y perspectivas mostraron lo siguiente: el género, el nivel educativo y la edad también dan cuenta de las diferencias evidenciadas en el acceso a los servicios financieros en la región. De hecho, el $35 \%$ de las mujeres de la región tienen una cuenta bancaria, mientras que en el caso de los hombres este porcentaje asciende a $44 \%$. A nivel mundial, estos porcentajes son de $47 \%$ para las mujeres y $55 \%$ para los hombres. La encuesta de Perú muestra que las mujeres tienen menos posibilidades de mantener un producto de pago frente a los hombres $(45 \%$ frente a $53 \%$ ). Asimismo, el nivel educativo también ayuda a explicar la variación en el uso de cuentas formales.

La variable cultura financiera aplicada en la encuesta de siete dimensiones, planteada y aplicada en la investigación de Mamani (2016), señala que estas dimensiones son "créditos, seguros, medios de pago, intereses, presupuesto, ahorro e inversión" (p. 30).

Luego de conceptualizar términos sobre cultura financiera, se tomó en cuenta sus respectivas competencias con la finalidad de adaptarlas a la encuesta, para determinar si los estudiantes de Administración del VI y VIII ciclo de ESAD y II Ciclo de ESCC de la UNJBG tienen conocimiento sobre la cultura financiera.

\section{OBJETIVOS}

\section{Objetivo General}

Determinar el nivel de conocimiento sobre la cultura financiera de los estudiantes del VI y VIII ciclo de ESAD y II ciclo de ESCCUNJBG.

\section{Objetivos Específicos}

1. Describir el nivel de conocimiento sobre créditos de los estudiantes del VI y VIII ciclo de ESAD y II de ESCC-UNJBG.

2. Analizar el nivel de conocimiento sobre seguros de los estudiantes del VI y VIII ciclo de ESAD y II de ESCC-UNJBG.

3. Determinar el nivel de conocimiento sobre medios de pago de los estudiantes del VI y VIII ciclo de ESAD y II de ESCC-UNJBG.

4. Analizar el nivel de conocimiento sobre interés de los estudiantes del VI y VIII ciclo de ESAD y II de ESCC-UNJBG.

5. Describir el nivel de conocimiento sobre presupuesto de los estudiantes del VI y VIII ciclo de ESAD y II de ESCC-UNJBG.

6. Determinar el nivel de conocimiento sobre ahorro de los estudiantes del VI y VIII ciclo de ESAD y II de ESCC-UNJBG.

7. Describir el nivel de conocimiento sobre inversión de los estudiantes del VI y VIII ciclo de ESAD y II de ESCC UNJBG.

\section{METODOLOGÍA}

La investigación se estructura dentro del enfoque cuantitativo, de nivel descriptivocomparativo y diseño no experimental, transversal (Hernández, Fernández y Baptista, 2014).

El método estadístico utilizado es el de población finita: 90 estudiantes (25 de II ciclo de ESCC, 33 de VI y 32 de VIII ciclo de ESADUNJBG).

La limitación es la no existencia de trabajos de investigación de cultura financiera en la escuela de profesional de comunicaciones 


\section{MATERIALES Y MÉTODOS}

Como técnicas de recolección de información se han utilizado la encuesta y la observación, los instrumentos empleados fueron la ficha de encuesta Tipo Likert con 24 ítems y la ficha de observación. Para el análisis de datos se utilizó SPSS 24.

El instrumento es altamente confiable, estimado por alfa Cronbach en 0.903 "correlacionados" (Welch \& Comer, 1988). Lo cual demuestra que es fiable y consistente.

\section{RESULTADOS}

Se han encuestado a 90 estudiantes (Tabla 1), donde 65 fueron de ESAD y 25 de la ESCC.

Tabla 1

Resumen de procesamiento de casos

\begin{tabular}{|llrr|} 
& & N & \% \\
\multirow{3}{*}{ Casos } & Válido & 90 & 100 \\
& Excluido & 0 & 0 \\
& Total & 90 & 100
\end{tabular}

Según el sexo del encuestado, $54.44 \%$ fueron de sexo masculino y $45.56 \%$ de sexo femenino (Figura 1).

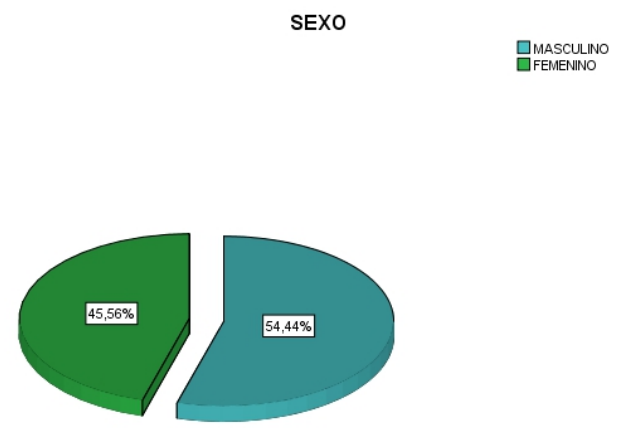

Figura 1. Sexo del encuestado (\%).

En lo que respecta a edades, estas oscilan entre $19-21$ años con un $48.89 \%$ y un $5.56 \%$ correspondiente a mayores de 25 años (Figura 2).

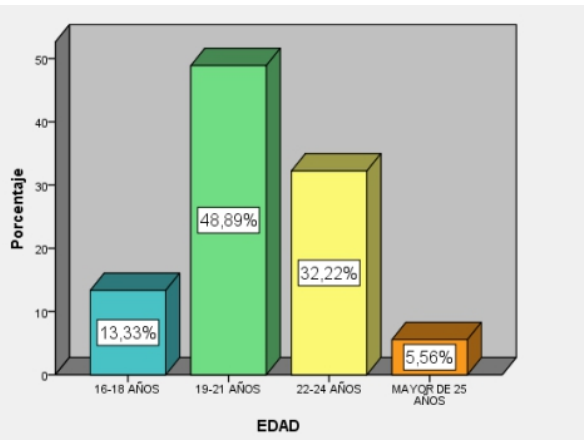

Figura 2. Edad del encuestado (\%).

Por otro lado, en la (Figura 3) muestra la procedencia de los encuestados, que en su mayor porcentaje pertenecen al Distrito Gregorio Albarracín (42.22\%).

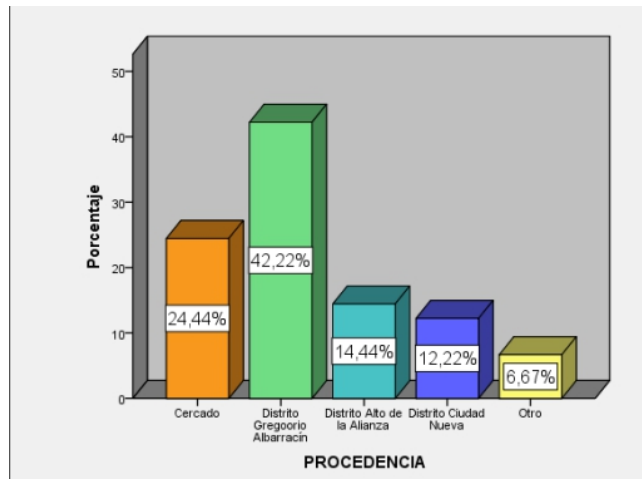

Figura 3. Procedencia del encuestado (\%).

Con respecto al ciclo de estudios, el $36.67 \%$ pertenecen al VI ciclo y el $35.56 \%$, al VIII de ESAD. Por otro lado, el $27.78 \%$ pertenecen al II de ESCC

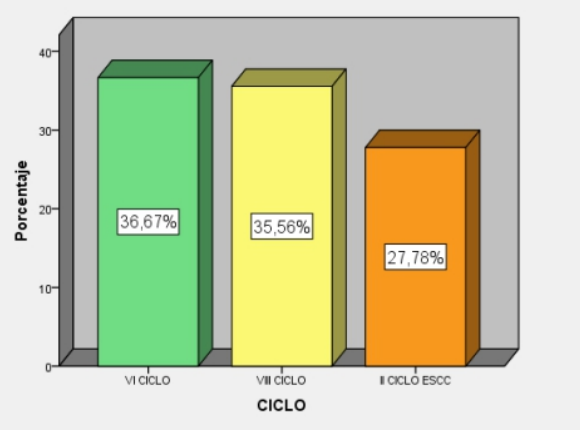

Figura 4. Ciclo de estudios del encuestado (\%). 
La participación por carrera profesional fue $72.22 \%$ de ESAD y un $27.78 \%$ de ESCC.

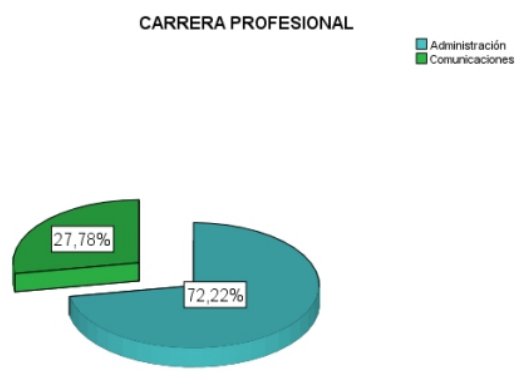

Figura 5. Carrera profesional del encuestado (\%).

Tabla 2

Cultura financiera

\begin{tabular}{|cccccc|}
\hline & Frecuencia & Porcentaje & $\begin{array}{c}\text { Porcentaje } \\
\text { válido }\end{array}$ & $\begin{array}{c}\text { Porcentaje } \\
\text { acumulado }\end{array}$ \\
\hline Válido & BAJO & 2 & 2.2 & 2.2 & 2.2 \\
& MEDIA & 20 & 22.2 & 22.2 & 24.4 \\
& ALTO & 68 & 75.6 & 75.6 & 100 \\
& Total & 90 & 100 & 100 & \\
\hline
\end{tabular}

Tabla 3

Cultura financiera ${ }^{*}$ Ciclo $\%$ del total

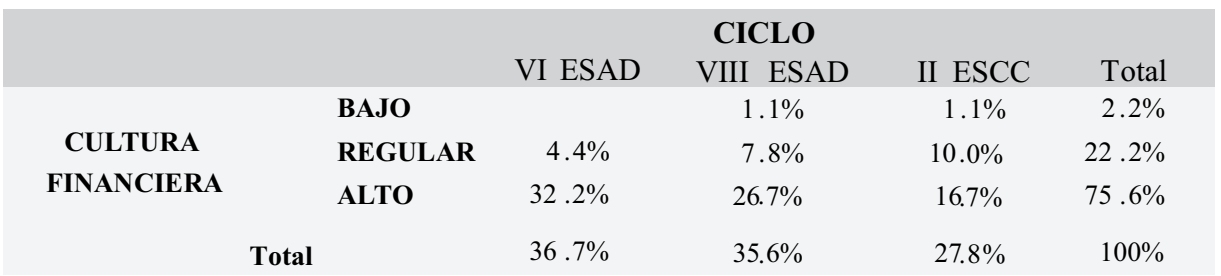

A continuación, se presentan los resultados obtenidos para determinar el nivel de conocimiento sobre las siguientes dimensiones: Créditos, Seguros, Medios de pago, Interés,

Presupuesto, Ahorro e Inversión de los estudiantes del VI y VIII ciclo de ESAD y II de ESCC-UNJBG. Respondiendo a los objetivos específicos.

\section{Nivel de conocimiento sobre Créditos de los estudiantes del VI y VIII ciclo de ESAD y II de ESCC-UNJBG: Dimensión 1. Créditos}

Tabla 4

Dimensión 1. Créditos

$\begin{array}{llcc} & & \text { Recuento } & \begin{array}{c}\text { \% de N } \\ \text { columnas }\end{array} \\ \text { P1. Consideras que es } & \text { Nunca/Totalmente en desacuerdo } & 4 & 4.4 \% \\ \text { necesario analizar un } & \text { Casi nunca/En desacuerdo } & 5 & 5.6 \% \\ \text { crédito, sea del tipo que sea, } & \text { A veces/Indeciso } & 14 & 15.6 \% \\ \text { hipotecario, personal, } & \text { Casi siempre/De acuerdo } & 19 & 21.1 \% \\ \text { comercial. } & \text { Siempre/Totalmente de acuerdo } & 48 & 53.3 \%\end{array}$


P2. Consideras que debes tener educación financiera sobre los créditos personales.

P3. Crees que debes ser disciplinado con el pago de los créditos.

P4. Consideras que las entidades financieras son transparentes en sus cálculos de tasa de interés.
Nunca/Totalmente en desacuerdo

Casi nunca/En desacuerdo

A veces/Indeciso

Casi siempre/De acuerdo

Siempre/Totalmente de acuerdo

Nunca/Totalmente en desacuerdo

Casi nunca/En desacuerdo

A veces/Indeciso

Casi siempre/De acuerdo

Siempre/Totalmente de acuerdo

Nunca/Totalmente en desacuerdo

Casi nunca/En desacuerdo

A veces/Indeciso

Casi siempre/De acuerdo

Siempre/Totalmente de acuerdo
$2.2 \%$

$5.6 \%$

$6.7 \%$

$31.1 \%$

$54.4 \%$

$3.3 \%$

$1.1 \%$

$7.8 \%$

$27.8 \%$

$60.0 \%$

$8.9 \%$

$34.4 \%$

$41.1 \%$

$8.9 \%$

$6.7 \%$

Tabla 5

Conocimiento sobre la dimensión crédito

\begin{tabular}{|c|c|c|c|c|c|}
\hline & & Frecuencia & Porcentaje & $\begin{array}{c}\text { Porcentaje } \\
\text { válido }\end{array}$ & $\begin{array}{l}\text { Porcentaje } \\
\text { acumulado }\end{array}$ \\
\hline \multirow{4}{*}{ Válido } & BAJO & 3 & 3.3 & 3.3 & 3.3 \\
\hline & MEDIO & 38 & 42.2 & 42.2 & 45.6 \\
\hline & ALTO & 49 & 54.4 & 54.4 & 100 \\
\hline & Total & 90 & 100 & 100 & \\
\hline
\end{tabular}

\section{Nivel de conocimiento sobre Seguros de los estudiantes del VI y VIII ciclo de ESAD y II de ESCC-UNJBG: Dimensión 2. Seguros}

Tabla 6

Dimensión 2. Seguros

\begin{tabular}{llcr} 
& & Recuento & \% \\
\hline $\begin{array}{l}\text { P5. Consideras que las } \\
\text { personas deben tener hábito } \\
\text { de seguro de vida, vivienda y } \\
\text { negocios. }\end{array}$ & Canca/Totalmente en desacuerdo & 2 & $2.2 \%$ \\
& A veces/Indeciso & 3 & $3.3 \%$ \\
& Casi siempre/De acuerdo & 12 & $13.3 \%$ \\
& Siempre/Totalmente de acuerdo & 43 & $47.8 \%$ \\
P6. Te prepararás para tener & Nunca/Totalmente en desacuerdo & 30 & $33.3 \%$ \\
$\begin{array}{l}\text { éxito en el manejo de los } \\
\text { riesgos. }\end{array}$ & A veces/Indeciso & 3 & $3.3 \%$ \\
& Casi siempre/De acuerdo & 9 & $10.0 \%$ \\
& Siempre/Totalmente de acuerdo & 22 & $24.4 \%$ \\
$\begin{array}{l}\text { P7. Consideras que una } \\
\text { buena cobertura de seguro } \\
\text { protege contra el riesgo y } \\
\text { garantiza que podamos } \\
\text { retenerbienes. }\end{array}$ & Casi nunca/En desacuerdo & 34 & $37.8 \%$ \\
& Casi siempre/De acuerdo & 22 & $24.4 \%$ \\
& Siempre/Totalmente de acuerdo & 3 & $3.3 \%$ \\
& & 5 & $5.6 \%$ \\
\end{tabular}


Tabla 7

Conocimiento sobre la dimensión seguros

\begin{tabular}{|cccccc|}
\hline & Frecuencia & Porcentaje & $\begin{array}{c}\text { Porcentaje } \\
\text { válido }\end{array}$ & $\begin{array}{c}\text { Porcentaje } \\
\text { acumulado }\end{array}$ \\
\hline \multirow{3}{*}{ Válido } & BAJO & 4 & 4.4 & 4.4 & 4.4 \\
& MEDIO & 59 & 65.6 & 65.6 & 70.0 \\
& ALTO & 27 & 30.0 & 30.0 & 100 \\
& Total & 90 & 100 & 100 & \\
\hline
\end{tabular}

\section{Nivel de conocimiento sobre Medios de pago de los estudiantes del VI y VIII ciclo de ESAD y II de ESCC-UNJBG: Dimensión 3. Medios de pago}

Tabla 8

Dimensión 3. Medios de pago

\begin{tabular}{|c|c|c|c|}
\hline & & Recuento & $\%$ \\
\hline \multirow{5}{*}{$\begin{array}{l}\text { P8. Utilizas el celular como } \\
\text { un medio de pago. }\end{array}$} & Nunca/Totalmente en desacuerdo & 20 & $22.2 \%$ \\
\hline & Casi nunca/En desacuerdo & 25 & $27.8 \%$ \\
\hline & A veces/Indeciso & 20 & $22.2 \%$ \\
\hline & Casi siempre/De acuerdo & 18 & $20.0 \%$ \\
\hline & Siempre/Totalmente de acuerdo & 7 & $7.8 \%$ \\
\hline \multirow{5}{*}{$\begin{array}{l}\text { P9. Cuando realizas tus } \\
\text { compras y/o ventas, el medio } \\
\text { de pago que utilizas son las } \\
\text { transferencias bancarias. }\end{array}$} & Nunca/Totalmente en desacuerdo & 12 & $13.3 \%$ \\
\hline & Casi nunca/En desacuerdo & 23 & $25.6 \%$ \\
\hline & A veces/Indeciso & 28 & $31.1 \%$ \\
\hline & Casi siempre/De acuerdo & 20 & $22.2 \%$ \\
\hline & Siempre/Totalmente de acuerdo & 7 & $7.8 \%$ \\
\hline \multirow{5}{*}{$\begin{array}{l}\text { P10 Consideras que debes } \\
\text { utilizar los medios } \\
\text { electrónicos para ser } \\
\text { competitivo a nivel nacional } \\
\text { e internacional. }\end{array}$} & Nunca/Totalmente en desacuerdo & 3 & $3.3 \%$ \\
\hline & Casi nunca/En desacuerdo & 7 & $7.8 \%$ \\
\hline & A veces/Indeciso & 14 & $15.6 \%$ \\
\hline & Casi siempre/De acuerdo & 35 & $38.9 \%$ \\
\hline & Siempre/Totalmente de acuerdo & 31 & $34.4 \%$ \\
\hline \multirow{5}{*}{$\begin{array}{l}\text { P11. Consideras que los } \\
\text { m e d i o s d e p a g o } \\
\text { tradicionales son necesarios } \\
\text { y son más seguros. }\end{array}$} & Nunca/Totalmente en desacuerdo & 5 & $5.6 \%$ \\
\hline & Casi nunca/En desacuerdo & 15 & $16.7 \%$ \\
\hline & A veces/Indeciso & 35 & $38.9 \%$ \\
\hline & Casi siempre/De acuerdo & 27 & $30.0 \%$ \\
\hline & Siempre/Totalmente de acuerdo & 8 & $8.9 \%$ \\
\hline
\end{tabular}

Tabla 9

Conocimiento sobre la dimensión medios de pago

\begin{tabular}{|cccccc|}
\hline & Frecuencia & Porcentaje & $\begin{array}{c}\text { Porcentaje } \\
\text { válido }\end{array}$ & $\begin{array}{c}\text { Porcentaje } \\
\text { acumulado }\end{array}$ \\
\hline \multirow{3}{*}{ Válido } & & & 7.8 & 7.8 \\
& BAJO & 7 & 7.8 & 82.2 & 90.0 \\
& MEDIO & 74 & 82.2 & 10.0 & 100 \\
& ALTO & 9 & 10.0 & 100 & \\
\hline
\end{tabular}




\section{Nivel de conocimiento sobre Interés de los estudiantes del VI y VIII ciclo de ESAD y II de ESCC-UNJBG: Dimensión 4. Interés}

Tabla 10

Dimensión 4. Interés

\begin{tabular}{|c|c|c|c|}
\hline & & Recuento & $\%$ \\
\hline \multirow{5}{*}{$\begin{array}{l}\text { P12. Crees debes conocer la } \\
\text { tasa de interés activa y } \\
\text { pasiva de entidades } \\
\text { financieras }\end{array}$} & Nunca/Totalmente en desacuerdo & 3 & $3.3 \%$ \\
\hline & Casi nunca/En desacuerdo & 5 & $5.6 \%$ \\
\hline & A veces/Indeciso & 6 & $6.7 \%$ \\
\hline & Casi siempre/De acuerdo & 31 & $34.4 \%$ \\
\hline & Siempre/Totalmente de acuerdo & 45 & $50.0 \%$ \\
\hline \multirow{5}{*}{$\begin{array}{l}\text { P13. Crees que debes } \\
\text { aprender a calcular la tasa de } \\
\text { interés y hacer las } \\
\text { comparaciones. }\end{array}$} & Nunca/Totalmente en desacuerdo & 0 & $0 \%$ \\
\hline & Casi nunca/En desacuerdo & 5 & $5.6 \%$ \\
\hline & A veces/Indeciso & 6 & $6.7 \%$ \\
\hline & Casi siempre/De acuerdo & 35 & $38.9 \%$ \\
\hline & Siempre/Totalmente de acuerdo & 44 & $48.9 \%$ \\
\hline \multirow{5}{*}{$\begin{array}{l}\text { P14. Consideras que es } \\
\text { importante para el } \\
\text { financiamiento de } 1 \\
\text { emprendimiento. }\end{array}$} & Nunca/Totalmente en desacuerdo & 1 & $1.1 \%$ \\
\hline & Casi nunca/En desacuerdo & 2 & $2.2 \%$ \\
\hline & A veces/Indeciso & 13 & $14.4 \%$ \\
\hline & Casi siempre/De acuerdo & 30 & $33.3 \%$ \\
\hline & Siempre/Totalmente de acuerdo & 44 & $48.9 \%$ \\
\hline
\end{tabular}

Tabla 11

Conocimiento sobre la dimensión tasa de interés

\begin{tabular}{|c|c|c|c|c|c|}
\hline & & Frecuencia & Porcentaje & $\begin{array}{c}\text { Porcentaje } \\
\text { válido }\end{array}$ & $\begin{array}{l}\text { Porcentaje } \\
\text { acumulado }\end{array}$ \\
\hline \multirow{4}{*}{ Válido } & BAJO & 5 & 5.16 & 5.6 & 5.6 \\
\hline & MEDIA & 30 & 33.3 & 33.3 & 38.9 \\
\hline & ALTO & 55 & 61.1 & 61.1 & 100 \\
\hline & Total & 90 & 100 & 100 & \\
\hline
\end{tabular}

\section{Nivel de conocimiento sobre Presupuesto de los estudiantes del VI y VIII ciclo de ESAD y II de ESCC-UNJBG: Dimensión 5. Presupuesto}

Tabla 12

Dimensión 5. Presupuesto

\begin{tabular}{|llcr} 
& & Recuento & \% \\
\hline $\begin{array}{l}\text { P15. Crees que la gestión del } \\
\text { presupuesto es la proyección }\end{array}$ & Nunca/Totalmente en desacuerdo & 0 & $0.0 \%$ \\
$\begin{array}{l}\text { y cuantificación de las metas } \\
\text { de ingresos y egresos }\end{array}$ & A veces/Indeciso & 3 & $3.3 \%$ \\
personales. & Casi siempre/De acuerdo & 17 & $18.9 \%$ \\
& Siempre/Totalmente de acuerdo & 37 & $41.1 \%$ \\
& & 33 & $36.7 \%$ \\
P16. Consideras que es una & Nunca/Totalmente en desacuerdo & 2 & $2.2 \%$ \\
herramienta indispensable & Casi nunca/En desacuerdo & 3 & $3.3 \%$ \\
para la gestión de una & A veces/Indeciso & 12 & $13.3 \%$ \\
empresa. & Casi siempre/De acuerdo & 33 & $36.7 \%$ \\
& Siempre/Totalmente de acuerdo & 40 & $44.4 \%$
\end{tabular}


P17. Consideras que el control debe ser a partir de los ingresos familiares con valores.

P18. Considero que se debe controlar el presupuesto general de la empresa.
Nunca/Totalmente en desacuerdo

Casi nunca/En desacuerdo

A veces/Indeciso

Casi siempre/De acuerdo

Siempre/Totalmente de acuerdo

Nunca/Totalmente en desacuerdo

Casi nunca/En desacuerdo

A veces/Indeciso

Casi siempre/De acuerdo

Siempre/Totalmente de acuerdo
$1.1 \%$

$5.6 \%$

$15.6 \%$

$47.8 \%$

$27 \quad 30.0 \%$

$1 \quad 1.1 \%$

$2 \quad 2.2 \%$

$13 \quad 14.4 \%$

$29 \quad 32.2 \%$

$45 \quad 50.0 \%$

Tabla 13

Conocimiento la dimensión presupuesto

\begin{tabular}{|cccccc|}
\hline & Frecuencia & Porcentaje & $\begin{array}{c}\text { Porcentaje } \\
\text { válido }\end{array}$ & $\begin{array}{c}\text { Porcentaje } \\
\text { acumulado }\end{array}$ \\
\hline \multirow{2}{*}{ Válido } & & & 3.3 & 3.3 \\
& BAJO & 3 & 3.3 & 26.7 & 30.0 \\
& MEDIO & 24 & 26.7 & 70.0 & 100 \\
& ALTO & 63 & 70.0 & 100 & \\
\end{tabular}

\section{Nivel de conocimiento sobre Ahorro de los estudiantes del VI y VIII ciclo de ESAD y II de ESCC-UNJBG: Dimensión 6. Ahorro}

Tabla 14

Dimensión 6. Ahorro

\begin{tabular}{|c|c|c|c|}
\hline & & Recuento & $\%$ \\
\hline \multirow[t]{5}{*}{$\begin{array}{l}\text { P19. Consideras que es } \\
\text { necesario ahorrar alguna } \\
\text { parte de los ingresos. }\end{array}$} & $\begin{array}{l}\text { Nunca/Totalmente en } \\
\text { desacuerdo }\end{array}$ & 5 & $5.6 \%$ \\
\hline & Casi nunca/En desacuerdo & 3 & $3.3 \%$ \\
\hline & A veces/Indeciso & 9 & $10.0 \%$ \\
\hline & Casi siempre/De acuerdo & 27 & $30.0 \%$ \\
\hline & $\begin{array}{l}\text { Siempre/Totalmente de } \\
\text { acuerdo }\end{array}$ & 46 & $51.1 \%$ \\
\hline \multirow[t]{5}{*}{$\begin{array}{l}\text { P20. Consideras que debes } \\
\text { trazar tus objetivos claros } \\
\text { para justificar el ahorro. }\end{array}$} & $\begin{array}{l}\text { Nunca/Totalmente en } \\
\text { desacuerdo }\end{array}$ & 2 & $2.2 \%$ \\
\hline & Casi nunca/En desacuerdo & 4 & $4.4 \%$ \\
\hline & A veces/Indeciso & 9 & $10.0 \%$ \\
\hline & Casi siempre/De acuerdo & 32 & $35.6 \%$ \\
\hline & $\begin{array}{l}\text { Siempre/Totalmente de } \\
\text { acuerdo }\end{array}$ & 43 & $47.8 \%$ \\
\hline \multirow{5}{*}{$\begin{array}{l}\text { P21. Crees que debes tener } \\
\text { e } 1 \text { hábito de a horro } \\
\text { examinado tus gastos. }\end{array}$} & $\begin{array}{l}\text { Nunca/Totalmente en } \\
\text { desacuerdo }\end{array}$ & 3 & $3.3 \%$ \\
\hline & Casi nunca/En desacuerdo & 0 & $0 \%$ \\
\hline & A veces/Indeciso & 6 & $6.7 \%$ \\
\hline & Casi siempre/De acuerdo & 42 & $46.7 \%$ \\
\hline & $\begin{array}{l}\text { Siempre/Totalmente de } \\
\text { acuerdo }\end{array}$ & 39 & $43.3 \%$ \\
\hline
\end{tabular}


Tabla 15

Conocimiento sobre la dimensión ahorro

\begin{tabular}{|c|c|c|c|c|c|}
\hline & & Frecuencia & Porcentaje & $\begin{array}{c}\text { Porcentaje } \\
\text { válido }\end{array}$ & $\begin{array}{l}\text { Porcentaje } \\
\text { acumulado }\end{array}$ \\
\hline \multirow{4}{*}{ Válido } & BAJO & 5 & 5.6 & 5.6 & 5.6 \\
\hline & MEDIO & 31 & 34.4 & 34.4 & 40.0 \\
\hline & ALTO & 54 & 60.0 & 60.0 & 100 \\
\hline & Total & 90 & 100 & 100 & \\
\hline
\end{tabular}

\section{Nivel de conocimiento sobre Inversión de los estudiantes del VI y VIII ciclo de ESAD y II de ESCC UNJBG: Dimensión 7. Inversión}

Tabla 16

Dimensión 7. Inversión

\begin{tabular}{|c|c|c|c|}
\hline & & Recuento & $\%$ \\
\hline \multirow{5}{*}{$\begin{array}{l}\text { P22. Consideras que debes } \\
\text { conocer sobre VAN, TIR } \\
\text { fin a n i e ros y otras } \\
\text { herramientas. }\end{array}$} & Nunca/Totalmente en desacuerdo & 0 & $0 \%$ \\
\hline & Casi nunca/En desacuerdo & 5 & $5.6 \%$ \\
\hline & A veces/Indeciso & 8 & $8.9 \%$ \\
\hline & Casi siempre/De acuerdo & 34 & $37.8 \%$ \\
\hline & Siempre/Totalmente de acuerdo & 43 & $47.8 \%$ \\
\hline \multirow{5}{*}{$\begin{array}{l}\text { P23. Crees que puedes } \\
\text { iniciarte estudiando en el } \\
\text { p r o y e c t o d e } \\
\text { emprendimiento en equipo. }\end{array}$} & Nunca/Totalmente en desacuerdo & 1 & $1.1 \%$ \\
\hline & Casi nunca/En desacuerdo & 2 & $2.2 \%$ \\
\hline & A veces/Indeciso & 10 & $11.1 \%$ \\
\hline & Casi siempre/De acuerdo & 36 & $40.0 \%$ \\
\hline & Siempre/Totalmente de acuerdo & 41 & $45.6 \%$ \\
\hline \multirow{5}{*}{$\begin{array}{l}\text { P24. Crees que se puede } \\
\text { aprovechar la oportunidad } \\
\text { invertir con nuevas ideas de } \\
\text { innovación. }\end{array}$} & Nunca/Totalmente en desacuerdo & 3 & $3.3 \%$ \\
\hline & Casi nunca/En desacuerdo & 1 & $1.1 \%$ \\
\hline & A veces/Indeciso & 7 & $7.8 \%$ \\
\hline & Casi siempre/De acuerdo & 29 & $32.2 \%$ \\
\hline & Siempre/Totalmente de acuerdo & 50 & $55.6 \%$ \\
\hline
\end{tabular}

Tabla 17

Conocimiento sobre la dimensión inversión

\begin{tabular}{|cccccc|}
\hline & Frecuencia & Porcentaje & $\begin{array}{c}\text { Porcentaje } \\
\text { válido }\end{array}$ & $\begin{array}{c}\text { Porcentaje } \\
\text { acumulado }\end{array}$ \\
\hline \multirow{3}{*}{ Válido } & BAJO & 4 & 4.4 & 4.4 & 4.4 \\
& MEDIO & 29 & 32.2 & 32.2 & 36.7 \\
& ALTO & 57 & 63.3 & 63.3 & 100 \\
& Total & 90 & 100 & 100 & \\
\hline
\end{tabular}




\section{DISCUSIÓN}

Con respecto al sexo del encuestado (54.44\% fueron de sexo masculino y $45.56 \%$ de sexo femenino) y a la edad el $48 \%$ oscilan entre 1921 años y el $5.56 \%$ son mayores de 25 años. Corroborando con anteriores investigaciones, por ejemplo, García, Grifoni, López, y Mejía (2013) sobre la educación financiera en América Latina y el Caribe, mostraron lo siguiente: El género, el nivel educativo y la edad también dan cuenta de las diferencias evidenciadas en el acceso a los servicios financieros en la región. De hecho, $35 \%$ de las mujeres de la región tienen una cuenta bancaria; mientras que en el caso de los hombres este porcentaje asciende a $44 \%$. A nivel mundial, estos porcentajes son de $47 \%$ para las mujeres y $55 \%$ para los hombres. La investigación tiene una relación con la UNJBG desde las autoridades y docentes en incentivar positivamente en la formación de estudiantes que conduzcan a ser profesionales con competencias y habilidades de la cultura financiera para la creación de valores con buen manejo de herramientas financieras.

La educación financiera puede contribuir a reducir las barreras a la demanda de inclusión financiera. Lo cual se corrobora con los estudios de López (2016), quien manifiesta que "la educación financiera puede aumentar el conocimiento y la comprensión de los productos y servicios financieros y, como tal, promover la demanda de los mismos, así como su uso efectivo". La investigación realizada por Aylas (2017) tiene relación indirecta con el presente trabajo de investigación, el autor señala que en su caso, el $78 \%$ de los estudiantes consideran que, si la persona tiene conocimientos sobre educación financiera tomará buenas decisiones en cuanto al uso de la tarjeta de crédito, por otro lado el conocimiento de temas financieros en los estudiantes se reconoce, ya que el $75 \%$ de ellos realizan presupuestos mensuales, lo cual demuestra que son capaces de afrontar en la práctica sus capacidades para tomar buenas decisiones.

El conocimiento de la cultura financiera es importante por las evidencias en los resultados obtenidos en las dimensiones de ahorro, crédito, presupuesto e inversión los cuales repercuten en formación de buenos gestores empresariales con valores y disciplina, además forma el hábito en lo personal, en la toma de decisiones e indirectamente favorece a la sociedad por creación de valor, crecimiento de las empresas y movimiento económico.

\section{Dimensión 1. Créditos}

En la Tabla 4 se observan 4 ítems, se destaca de ellos lo siguiente:

P1. Si considera necesario analizar un crédito, sea del tipo que sea, los resultados fueron: el $53.3 \%$ consideró que siempre y un $4.4 \%$, nunca.

P2. Si considera que se debe tener educación financiera sobre los créditos personales: el 54.4 $\%$ señaló que siempre y un $2.2 \%$, nunca.

P3. Si considera que se debe ser disciplinado con el pago de los créditos: El $60 \%$ señaló que siempre y un $3.3 \%$, nunca.

P4. Si considera que las entidades financieras son transparentes en sus cálculos de tasa de interés: el $41.15 \%$ considera que $a$ veces y un $34.4 \%$, casinunca.

A nivel de dimensión conocimiento sobre créditos, de los 90 estudiantes encuestados, 49 $(54.4 \%)$ obtuvieron un nivel alto y $3(3.3 \%)$, un nivel bajo (Tabla 5).

\section{Dimensión 2. Seguros}

En la Tabla 6 se observan 3 ítems, se destaca de ellos lo siguiente:

P5. Si considera que las personas deben tener hábito de seguro de vida, vivienda y negocios: $47.8 \%$ considera que casi siempre y $2.2 \%$, nunca.

P6. Si se prepara para tener éxito en el manejo de los riesgos: el $37.8 \%$ señala casi siempre y $3.3 \%$ nunca.

P7. Si considera que un seguro protege contra el riesgo y garantiza que podamos retener bienes: el $50 \%$ manifiesta casi siempre y $3.3 \%$, nunca.

A nivel de dimensión conocimiento sobre seguros, de 90 estudiantes encuestados, 59 (65.6\%) obtuvieron un nivel medio y $4(4.4 \%)$, un nivel bajo (Tabla 7). 


\section{Dimensión 3. Medios de pago}

En la Tabla 8 se observan 3 ítems y resaltamos de ellos lo siguiente:

P8. Si utiliza el celular como un medio de pago: el $20 \%$ mencionó que casi siempre y $22.2 \%$, nunca.

P9. El medio de pago que utilizó son las transferencias bancarias: el $22.2 \%$ manifestó que casi siempre y $13.3 \%$, nunca.

P10. Si considera que debe utilizar los medios electrónicos para ser competitivo a nivel nacional e internacional: el $38.9 \%$ considera que casi siempre y $3.3 \%$, nunca.

P11. Si considera que los medios de pago tradicionales son necesarios y son más seguros: el $30 \%$ casi siempre y $5.6 \%$, nunca.

A nivel de dimensión conocimiento sobre medios de pago, de 90 estudiantes encuestados, $74(82.2$. \%) obtuvieron un nivel alto y 7 (7.8 $\%$ ), un nivel bajo (Tabla 9).

\section{Dimensión 4. Interés}

En la Tabla 10 se observan 4 ítems y se resumen de esta lo siguiente:

$\mathrm{P} 12$. Si cree que debe conocer la tasa de interés activa y pasiva de entidades financieras: $50 \%$ considera que siempre y $3.3 \%$, nunca.

P13. Si debe aprender a calcular la tasa de interés y hacer las comparaciones: $48.9 \%$ cree que siempre y $5.6 \%$, a veces.

P14. Si se considera importante para el financiamiento del emprendimiento: $48.9 \%$ señala que siempre y $1.1 \%$, nunca.

A nivel de dimensión conocimiento sobre interés, de 90 estudiantes encuestados, 55 (61.1 $\%$ ) obtuvieron un nivel alto y $5(5.6 \%)$, un nivel bajo (Tabla 11).

Sobre esto tema, más conocimientos podrían ayudar a los estudiantes en este tema, por ejemplo, Blacio (2016) muestra de manera práctica como evidenciar la tasa de interés nominal calculándolo mediante la fórmula matemática respectiva y poder compararlo con las ofertas de inversión que se nos presente, teniendo la certeza de que nuestra elección será la más conveniente y la que nos genere mayor rentabilidad.

\section{Dimensión 5. Presupuesto}

En la Tabla 12 se observan 4 ítems y resaltamos de ellos lo siguiente:

P15. Si cree que la gestión del presupuesto es la proyección y cuantificación de las metas de ingresos y egresos personales: $41.1 \%$ considera que casi siempre y $0.0 \%$, nunca.

P16. Si considera que es herramienta indispensable para la gestión de una empresa: $44.4 \%$ opina que siempre y $2.2 \%$, nunca.

P17. Si considera que el control debe partir de los ingresos familiares con valores: $47.8 \%$ señala que casi siempre y $1.1 \%$, nunca.

P18. Si considera que debe controlar el presupuesto general de la empresa: el $50 \%$ opina que siempre y $1.1 \%$, nunca.

A nivel de dimensión conocimiento sobre presupuesto, de 90 estudiantes encuestados, 63 (70.0\%) obtuvieron un nivel alto y $3(3.3 \%)$, un nivel bajo (Tabla 13).

Por lo antes mencionado, Aguilar y Ortiz (2013) manifiestan que el conocimiento financiero es importante, pero lo es más la adquisición de hábitos financieros. En la investigación podemos determinar que en el aspecto de planeación y control financiero no se logra generar una aplicación en la vida de los estudiantes que garantice el buen manejo de sus finanzas personales y se complementen con la importancia de materias como finanzas, presupuestos y matemáticas financieras.

\section{Dimensión 6. Ahorro}

En la Tabla 14 se observan 3 ítems y resaltamos de ellos lo siguiente:

P19. Considera que debe ahorrar alguna parte de los ingresos: $51.1 \%$ opina que siempre y 5.6 $\%$, nunca.

P20. Considera que debe trazar mis objetivos claros para justificar el ahorro: $47.8 \%$ señala que siempre y $2.2 \%$, nunca.

P21. Considera que debe tener el hábito de ahorro examinado gastos: $46.7 \%$ manifiesta que casi siempre y $3.3 \%$, nunca.

A nivel de dimensión conocimiento sobre ahorro, de 90 estudiantes encuestados, 54 (60 $\%$ ) obtuvieron un nivel alto y $5(5.6 \%)$, un nivel bajo (Tabla 15). 
Esta es una de las dimensiones que más interés nos debería provocar. Según autores como Larios (2009), la práctica del ahorro debe ser prioritaria para el desarrollo de habilidades de la organización, control y evaluación para el logro de objetivos y metas establecidas tanto individual y colectivamente. Por ello, es importante considerar la cultura del ahorro como una herramienta que permitirá, a los estudiantes de la UNJBG, el cumplimiento de logros al mediano y largo plazo. Los ahorros en el seno de los hogares son debido a buenos hábitos inculcados por los padres conductores de las familias sumado los valores sociales (la universidad) se convierten entonces en la práctica aceptada de buenos hábitos en el hogar y en beneficio de la comunidad.

\section{Dimensión 7. Inversión}

En la Tabla 16 se observan 3 ítems y resaltamos de ellos lo siguiente:

P22. Considera que debe conocer sobre VAN, TIR financieros y otras herramientas: $47.8 \%$ señaló que siempre y $0 \%$, nunca.

$\mathrm{P} 23$. Considera que puede iniciar en el proyecto de emprendimiento en equipo $45.6 \%$ manifestó que siempre y $1.1 \%$, nunca.

P24. Considera que puede aprovechar la oportunidad invertir con nuevas ideas de innovación $55.6 \%$ siempre y $1.1 \%$, nunca.

A nivel de dimensión conocimiento sobre inversión, de 90 estudiantes encuestados, 57 (63.3\%) obtuvieron un nivel alto y $4(4.4 \%)$, un nivel bajo (Tabla 17).

No cabe duda que el conocimiento sobre la cultura financiera con valores es importante en los estudiantes de educación superior porque habilita de competencias en la formación profesional. Además, es de suma utilidad en el ámbito personal, contribuyendo a ser competitivo en la toma de decisiones en la gestión empresarial en un mercado de incertidumbre y de economía globalizada, garantizada por el aprendizaje de herramientas financieras en la creación de valor.

\section{CONCLUSIONES}

Sobre el nivel de conocimiento de los estudiantes del VI y VIII ciclo de ESAD y II de ESCC de la UNJBG sobre la cultura financiera se concluye que, de los 90 estudiantes, un 75.6 $\%$ han alcanzado un nivel alto en conocimiento sobre las dimensiones de: crédito, seguros, medios de pago, tasa de interés, presupuesto, ahorro e inversión.

En la descripción de conocimiento sobre la dimensión crédito se concluye que, de los 90 estudiantes, un $54.4 \%$ han alcanzado un nivel alto en conocimiento de la necesidad de análisis de crédito, educación financiera, disciplina en los pagos, y transparencia en la tasa de interés.

En cuanto a la dimensión de seguros se concluye que, de los 90 estudiantes, un $65.6 \%$ han alcanzado un nivel regular en conocimiento en la necesidad de formación de hábitos de seguro, manejo de riesgos y cobertura de seguro.

En la descripción sobre la dimensión medios de pago se concluye que, de los 90 estudiantes, un $82.2 \%$ han alcanzado un nivel regular de conocimiento en el uso de celular, transferencias bancarias, medios electrónicos y medios tradicionales.

Sobre la dimensión tasa de interés, se concluye que, de los 90 estudiantes, un $61.1 \%$ han alcanzado un nivel alto en la necesidad de conocimiento en el uso de tasa de interés, aprendizaje de cálculo, comparaciones y la utilidad como herramienta de financiamiento de emprendimiento.

Respecto a la dimensión presupuesto se concluye que, de los 90 estudiantes, un $70 \%$ han alcanzado un nivel alto en la necesidad de conocimiento sobre gestión de presupuesto y consideran que es una herramienta de gestión empresarial, necesidad de control de ingresos con valores y control del presupuesto de la empresa.

En el caso del conocimiento sobre la dimensión ahorro se concluye que, de los 90 estudiantes un $60 \%$ han alcanzado un nivel alto en la necesidad de conocimiento sobre ahorrar parte 
de los ingresos, tener objetivos claros en el ahorro y formar un hábito de ahorro.

Finalmente, sobre en los hallazgos sobre el conocimiento de la dimensión de inversión, se concluye que, de los 90 estudiantes, un $63.3 \%$ han alcanzado un nivel alto en la necesidad de conocimiento de indicadores y herramientas financieras, sobre iniciar en proyecto de emprendimiento en equipo y aprovechar oportunidades de invertir con nuevas ideas de innovación.

\section{REFERENCIAS BIBLIOGRÁFICAS}

Aguilar, X. y Ortiz, B. (2013). Diseño de un programa de educación y cultura financiera para los estudiantes de modalidad presencial de la titulación en administración en banca y finanzas, 2013 (tesis de pregrado). Universidad técnica particular de Loja. Ecuador.

Ardila, Y., y Rengifo, L. (2014). Aproximaciones a la educación financiera en América Latina. Llevado a cabo en el XIX Congreso Internacional de Contaduría, Administración e Informática, México, D.F.

Aylas, Y. (2017). Análisis de la relación entre la educación financiera y variables de la tarjeta de crédito en los estudiantes de la modalidad presencial de la Facultad de Ciencias de la Empresa de la Universidad Continental - sede Huancayo, 2017 (tesis de pregrado). Universidad Continental, Huancayo.

Blacio, K. (2016). Cálculo de la tasa nominal que realizan las personas y las empresas en inversiones financieras. Machala, Ecuador: Universidad Técnica de Machala

Blancas, Y. (2016). La cultura financiera y su impacto en la gestión crediticia de la agencia el Tambo de la CMAC Huancayo en el año 2014 (tesis de pregrado). Universidad Peruana Los Andes, Huancayo, Perú.
Briano, G., Quevedo, L. y Castañón, E. (2016). Midiendo la cultura financiera en estudiantes universitarios: El caso de la Facultad de Contaduría y Administración de la UASLP. Universidad Autónoma de San Luis Potosí. $\mathrm{R} \mathrm{e} \mathrm{c} \mathrm{u} \mathrm{p} \mathrm{e} \mathrm{r} \mathrm{a} \mathrm{d} \mathrm{o} \mathrm{d} \mathrm{e}$ https://riica2016.unican.es/wpcontent/uploads/2016/06/RIICA -2016-Sesiones-ParalelasMesa-5-Comunicaciones.pdf

Cabrera, E., y Zapata, J. (2017). Cultura financiera de los estudiantes de la Universidad Tecnológica Metropolitana. Universidad Tecnológica Metropolitana. Advances in Engineering and Innovation. 2(3), 20-33. $\mathrm{R}$ e c u p e r a d o d e congreso.investiga.fca.unam.m $\mathrm{x} / \mathrm{docs} / \mathrm{xix} / \mathrm{docs} / 9.04 . \mathrm{pdf}$

Fagilde, C. (2009). Presupuesto empresarial. Un enfoque práctico para el aula. Barinas: Programa de Ciencias Sociales.

Flores, S. (2018). Implicancia de la cultura financiera en la capacidad de ahorro de los clientes de la caja Arequipa agencia Puerto Maldonado, período 2016 (tesis de pregrado). Universidad Andina del Cusco

García, F. (2017). El crédito comercial. Importancia de esta modalidad de financiación en Europa y los efectos de la crisis. Recuperado $\mathrm{d}$

https://repositorio.unican.es/xm lui/handle/10902/12726

García, N., Grifoni, A., López, J. y Mejía, D. (2013). La educación financiera en América Latina y el Caribe. Situación actual y perspectivas. CAF.

Hernández, R., Fernández, C. y Baptista, P. (2014). Metodología de la investigación. México: McgrawHill.

Larios, J. (2009). Ahorro Futuro, Educar en la Protección del Medio Ambiente. Hekademus: Revista Cientifica 
de la Fundación Iberoamericana para la Excelencia Educativa, (4), 1827.

Lobato, T., Santillán, A. y Hernández, J. (2018). Algunas características que explican el comportamiento de los jóvenes universitarios hacia el ahorro. International Journal of Developmental and Educational Psychology. Revista INFAD de Psicología., 1(2), 159-170. Recuperado de http://www.infad.eu/RevistaINF AD/OJS/index.php/IJODAEP/a rticle/view/1141

Lopez, J. (2016). La (Des) educación Financiera en Jóvenes Universitarios Ecuatorianos: Una Aproximación Teórica. Revista Empresarial, ICE-FEE$U C S G, 10(1), 6-41$. $\mathrm{R}$ e c u p e r a d o d e https://dialnet.unirioja.es/descar ga/articulo/5580338.pdf

Lusardi, A., Mitchell, O. \& Curto, V. (2010). Financial literacy among the young. Journal of consumer affairs, 44(2), 358-380. doi: $10.3386 / \mathrm{w} 15352$

Mamani, N. (2016). El nivel de cultura financiera en los comerciantes del mercado internacional San José de la ciudad de Juliaca base II y III-2015 (tesis de pregrado). Universidad Peruana Unión, Juliaca, Perú.

García, V. (2014). Introducción a las finanzas. México: Grupo Editorial Patria.

Quintero, S. (2014). Educación económica y financiera en Colombia desde la perspectiva del desarrollo humano (tesis doctoral). Universidad Santo Tomás. Bogotá, Colombia.

Tinoco, W. (2017). Educación financiera en estudiantes universitarios de una universidad del departamento de Junín - 2017 (tesis de maestría). Universidad César Vallejo, Perú.

Welch, S. \& Comer, J. (1988). Quantitative Methods for Public Administration: Techniques And Applications. 